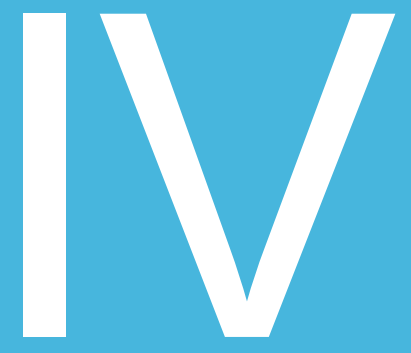

\title{
Conocimientos y actitudes sobre el aborto inducido en adolescentes del 5 to año de secundaria de una institución educativa
}

Knowledge and attitudes on induced abortion in adolescents 5th year of high School

Universidad Peruana Unión, Perú

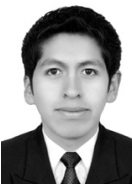

\section{Grover Eyden Trujillo Mamani}

Licenciado en enfermería por la Universidad Peruana Unión. Realizó diferentes cursos de actualización y especialización con especial enfoque en investigación y estadística.

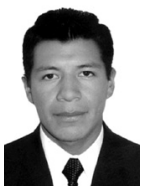

\section{Efrain Sembrera Farceque}

Licenciado en Enfermería por la Universidad Peruana Unión. Actualmente viene estudiando una segunda especialidad en promoción de la salud en la Facultad de Enfermería de la Universidad Nacional del Altiplano - Puno 
Grover Eyden Trujillo Mamani -

Efrain Sembrera Farceque

\section{Resumen}

El objetivo de esta investigación fue determinar la relación entre conocimientos y actitudes hacia el aborto inducido en adolescentes del quinto año. El diseño de investigación es no experimental de corte transversal de tipo correlacional, se realizó un muestreo probabilístico estratificado, determinándose una muestra de 128 estudiantes matriculados en el periodo académico 2015. El instrumento que se utilizó es el cuestionario de conocimientos sobre aborto inducido que consta de 14 preguntas y la escala de actitudes hacia el aborto inducido que consta de 18 ítems con respuesta en la escala de Likert, tiene como duración de 15 minutos. Los resultados indican que el $53.9 \%$ de los estudiantes presentan un nivel medio de conocimientos sobre el aborto inducido y solamente el $22.7 \%$ presenta un nivel alto; con respecto a las actitudes el $43.0 \%$ de los estudiantes presenta una actitud de aceptación hacia el aborto inducido y el $21.9 \%$ presentan una actitud de rechazo hacia el aborto, encontrándose correlación significativa entre las variables de estudio (sig=.000). Conclusiones: Mientras más alto el conocimiento mayor actitud de rechazo hacia el aborto inducido y mientras más bajo es el nivel de conocimientos mayor actitud de aceptación hacia el aborto inducido.

Palabras clave: Conocimiento, actitudes, aborto, aborto inducido.

\section{Abstract}

The object of this study is to determine the relation between knowledge and attitude about induced abortion in 5th year high school teen. The research design is not experimental with a cross-sectional, correlational type. A stratified probability sampling was conducted, determining a sample of 128 students enrolled in the academic year of 2015. The instrument used is the questionnaire of knowledge about induced abortion consisting of 14 questions and the scale of attitudes about induced abortion consisting of 18 items with a response in the Likert scale, it has a duration of 15 minutes. The results indicate that $53.9 \%$ of students have an average level of knowledge about induced abortion and just $22.7 \%$ have a high level; in relation to the attitudes $43.0 \%$ of students present an attitude of acceptance for induced abortion and $21.9 \%$ present and attitude of rejection for abortion. A significant correlation was found between the study variables (sig = $.000)$. Conclusions: the higher the knowledge, the higher the attitude of rejection for induced abortion; the lower the level of knowledge, the higher the attitude of acceptance for induced abortion.

Keywords: Knowledge, attitudes, abortion, induced abortion. 


\section{Introducción}

Actualmente es notable observar que los adolescentes sienten desorientación, confusión, poca seguridad y confianza en sí mismos; convirtiéndolos en un grupo vulnerable a desarrollar conductas sexuales de riesgo, tales como: el inicio precoz de actividades sexuales, conllevándolos a infecciones de transmisión sexual, VIH - SIDA, embarazos no planificados y abortos; poniendo en riesgo su salud sexual y reproductiva.

Según la Organización Mundial de la Salud (OMS) se calcula que cada año se realizan unos 22 millones de abortos peligrosos en todo el mundo, casi todos ellos en países en desarrollo, las defunciones debidas a abortos peligrosos representan el $13 \%$ de todas las defunciones maternas. África se ve desproporcionalmente afectada, por cuanto dos tercios de todas las defunciones relacionadas con el aborto tienen lugar en ese continente (OMS, 2014).

En todo el mundo, anualmente, aproximadamente 15 millones de adolescentes de 15 a 19 años dan a luz, representando más de $10 \%$ de los nacimientos. Por lo menos 1,25 millones quedan embarazadas a cada año en los 28 países de la Organización para Cooperación y Desarrollo Económico (OCDE). De estas, cerca de medio millón procura la realización del aborto. En América Latina, los datos sobre el embarazo en la adolescencia han mostrado un aumento en la tasa de fecundidad para esta población cuando comparada a mujeres adultas, especialmente en los países más pobres (Ribeiro da Fonseca, et al., 2013).

En el Perú en las últimas décadas el embarazo adolescente (entre 15 a 19 años) de manera global se encuentra entre el $12 \%$ y $13 \%$, llegando a valores del $21.5 \%$ en las zonas rurales, $12.4 \%$ en la sierra, $27.5 \%$ en la selva; y con respecto al nivel de educación, el $56.7 \%$ de los adolescentes embarazados no tienen educación, el $34.9 \%$ presentan educación primaria, el $11.6 \%$ educación secundaria y el $4.5 \%$ educación superior. Con respecto a la iniciación sexual en mujeres adolescentes existe un aumento sostenible en los últimos 20 años sobre el inicio de la primera relación sexual antes de los 15 años, siendo este del $6 \%$, y el $50.6 \%$ de los adolescentes usan métodos anticonceptivos (Mendoza \& Subiria, 2013).

En Arequipa, del total del número de embarazos en adolescentes que se registran al año en Arequipa, entre el 10 y $15 \%$ terminan en abortos, producto de relaciones sexuales prematuras y prácticas abortivas ilegales, señaló el médico Luis Alberto Bernal; además, indicó que los abortos se producen porque en nuestro país no existe una adecuada educación sexual que debe ser brindada por la familia y la escuela, y por el escaso conocimiento sobre los métodos anticonceptivos (La República, 2004) y de acuerdo al reporte del Ministerio de Salud, Arequipa es la 
Grover Eyden Trujillo Mamani -

Efrain Sembrera Farceque

tercera ciudad del país donde se realizan abortos clandestinos (Radio Programas del Perú, 2011).

A nivel de la región, la Red de Salud de San Román ocupa el primer lugar en presentar abortos, con 65 casos reportados sólo en dos meses, enero y febrero, según lo dio a conocer María Condori Apaza, obstetra del hospital Carlos Monge Medrano de Juliaca. Del $100 \%$ de abortos presentados en San Román, la mitad corresponden a menores de edad, una práctica que se realiza durante los tres primeros meses de gestación (Condori, 2013), además señaló que en el 2012 se registraron 1200 casos de abortos, de los cuales un aproximado de 119 se practicaron a mujeres de 18 a 29 años; 23 de ellas, adolescentes de 12 a 17 años, en quienes existía el riesgo de una hemorragia interna, ya que muchas veces la placenta no se desprende con normalidad.

Debido a que las cifras estadísticas sobre la práctica del aborto en América Latina y el Perú, muestran; que el número de abortos se encuentra en incremento; el aborto inducido actualmente es reconocido como un problema de Salud Pública, por su magnitud, la gravedad de sus consecuencias y su repercusión psicológica, social y económica. Son relativamente escasos los intentos que se han hecho por identificar los conocimientos; es decir, la información que tienen sobre el tema, asimismo la actitudes hacia ésta problemática.

La presente investigación se centra fundamentalmente en los adolescentes porque son un grupo vulnerable a desarrollar conductas sexuales de riesgo; puesto que en su formación como individuo acceden a temas sobre salud sexual y reproductiva pero de manera limitada y muchas veces errónea, por lo que la información que adquieren son insuficientes, que posteriormente afecta negativamente en la toma de decisiones respecto a su vida sexual, favoreciendo el embarazo no deseado en los mismos, y despertando de esta manera la tentativa del aborto con el fin de remediar los problemas originados, sin conocer, ni asumir las consecuencias que posteriormente pueden afectar de manera significativa sobre la salud y su bienestar.

Este estudio se justifica por la relevancia que el mismo presenta desde el punto de vista teórico, práctico, económico y social; y finalmente se justifica por los aportes realizados a las líneas de investigación desarrolladas por la Escuela Profesional de Enfermería de la Universidad Peruana Unión: Salud y desarrollo comunitario.

El objetivo general de la investigación fue determinar la relación entre conocimientos y actitudes hacia el aborto inducido en adolescentes del quinto año de educación secundaria de la Gran Unidad Escolar Las Mercedes, Juliaca - 2015. 


\section{Materiales y métodos}

\subsection{Participantes}

La presente investigación se realizó con los estudiantes del 5to grado de educación secundaria de la I.E. Gran Unidad Escolar Las Mercedes de la ciudad de Juliaca, matriculados en el año lectivo 2015, la población de estudio es de ambos sexos, las edades oscilan entre 15 y 18 años; la institución educativa se encuentra ubicada en el departamento de Puno - Perú, Provincia de San Román, Juliaca.

La población está conformada por 384 estudiantes distribuidos en 10 secciones. Para la determinación de la muestra, se realizó un muestreo probabilístico estratificado, quedando la muestra en 128 estudiantes.

\subsection{Instrumentos}

Según Valderrama (2002) se utilizó la técnica de la encuesta, aplicando los cuestionarios sobre conocimientos sobre aborto inducido y el cuestionario de actitudes frente al aborto inducido.

El instrumento que valora conocimiento está conformado por 14 preguntas, distribuidos en tres secciones (conocimientos generales, causas y consecuencias) las preguntas son de selección múltiple, donde cada pregunta correcta se valora con un punto y las incorrectas con cero puntos, se utilizó una escala evaluativa de acuerdo a tres categorías que son: bajo, medio y alto, según la puntuación obtenida por cada adolescente, el instrumento fue sometido a juicio de expertos, con la participación de ocho profesionales con experiencia en el tema de investigación y obtuvo una confiabilidad de 0.74 .

El instrumento que valora las actitudes para la recolección de datos es la escala de Lickert modificada con 18 enunciados referentes a las actitudes de los adolescentes hacia el aborto inducido, cada ítem es evaluado con las siguientes escalas: Totalmente de Acuerdo (TA), Acuerdo (A), Indiferente (I), Desacuerdo (D) y Totalmente desacuerdo (TDA), cada ítems positivo se valora de 5 puntos a 1 punto, y los ítems negativos la calificación va de 1 punto para totalmente de acuerdo hasta 5 puntos para totalmente en desacuerdo; los ítems positivos son el $1,3,4,7,10,11,14,15$ y 17 y los ítems negativos son: $2,5,6,8,9,12,13,16$ y 18. Los análisis de fiabilidad obtenidos fueron de 62. Elaborados por Palomino (2009).

La información se recogió previa autorización de la Institución Educativa, así mismo se informó a los estudiantes sobre el propósito, los objetivos y el anonimato de la información. 
Grover Eyden Trujillo Mamani -

Efrain Sembrera Farceque

\subsection{Análisis de datos}

El presente estudio corresponde al tipo de investigación correlacional, Se utiliza cuando se tiene como propósito conocer la relación existente entre dos o más conceptos, categorías o variables en un contexto en particular (Hernandez, Fernández, \& Baptista, 2010).

Según Hernández et al. (2010) y Valderrama (2002) el diseño de la investigación es no experimental de corte transversal; ya que no existe manipulación activa de alguna variable y se busca establecer la relación de variables medidas en una muestra en un único momento del tiempo.

Para el procedimiento de datos se realizó mediante la consistencia del análisis de la información y tabulación de datos. Para el análisis de datos se utilizó el paquete estadístico SPSS, versión 21.0 en español.

Los datos se presentan mediante tablas de distribución de frecuencias y la hipótesis se probó mediante el estadístico del chi cuadrado.

\section{Resultados y Discusión}

\subsection{Resultados}

\section{En relación al objetivo general}

\section{Tabla 1}

Tabla de contingencia entre conocimientos y actitudes hacia el aborto inducido que presentan los adolescentes del quinto año de educación secundaria de la Gran Unidad Escolar Las Mercedes de la ciudad de Juliaca - 2015.

\begin{tabular}{|c|c|c|c|c|c|c|}
\hline & & & \multicolumn{3}{|c|}{ Conocimiento } & \multirow{2}{*}{ Total } \\
\hline & & & Bajo & Medio & Alto & \\
\hline \multirow{9}{*}{ Actitud } & \multirow{4}{*}{$\begin{array}{l}\text { Actitud de } \\
\text { aceptación }\end{array}$} & Recuento & 23 & 31 & 1 & 55 \\
\hline & & $\%$ de Actitud & $41.8 \%$ & $56.4 \%$ & $1.8 \%$ & $100.0 \%$ \\
\hline & & $\%$ de Conocimiento & $76.7 \%$ & $44.9 \%$ & $3.4 \%$ & $43.0 \%$ \\
\hline & & $\%$ del total & $18.0 \%$ & $24.2 \%$ & $.8 \%$ & $43.0 \%$ \\
\hline & \multirow{4}{*}{$\begin{array}{l}\text { Actitud de } \\
\text { indiferencia }\end{array}$} & Recuento & 4 & 28 & 13 & 45 \\
\hline & & $\%$ de Actitud & $8.9 \%$ & $62.2 \%$ & $28.9 \%$ & $100.0 \%$ \\
\hline & & $\%$ de Conocimiento & $13.3 \%$ & $40.6 \%$ & $44.8 \%$ & $35.2 \%$ \\
\hline & & $\%$ del total & $3.1 \%$ & $21.9 \%$ & $10.2 \%$ & $35.2 \%$ \\
\hline & \multirow{8}{*}{$\begin{array}{l}\text { Actitud de } \\
\text { rechazo }\end{array}$} & Recuento & 3 & 10 & 15 & 28 \\
\hline & & $\%$ de Actitud & $10.7 \%$ & $35.7 \%$ & $53.6 \%$ & $100.0 \%$ \\
\hline & & $\%$ de Conocimiento & $10.0 \%$ & $14.5 \%$ & $51.7 \%$ & $21.9 \%$ \\
\hline & & $\%$ del total & $2.3 \%$ & $7.8 \%$ & $11.7 \%$ & $21.9 \%$ \\
\hline \multirow[t]{4}{*}{ Total } & & Recuento & 30 & 69 & 29 & 128 \\
\hline & & $\%$ de Actitud & $23.4 \%$ & $53.9 \%$ & $22.7 \%$ & $100.0 \%$ \\
\hline & & $\%$ de Conocimiento & $100.0 \%$ & $100.0 \%$ & $100.0 \%$ & $100.0 \%$ \\
\hline & & $\%$ del total & $23.4 \%$ & $53.9 \%$ & $22.7 \%$ & $100.0 \%$ \\
\hline
\end{tabular}

Fuente: Encuesta de conocimiento y actitudes a los estudiantes de la I.E. G.U.E.L.M. 
En relación al nivel de conocimientos la mayoría de los estudiantes presentan un nivel medio de conocimientos (53.9\%), seguido de los niveles bajo (23.4\%) y alto (22.7\%); en relación a las actitudes hacia el aborto, se aprecia que la mayoría de los estudiantes presentan una actitud de aceptación del aborto inducido (43.0\%), seguido de las actitudes de indiferencia (35.2\%) y actitud de rechazo $(21.9 \%)$.

Así mismo el $24.2 \%$ de los estudiantes presentan un nivel de conocimientos medio y actitud de aceptación hacia el aborto inducido, el $21.9 \%$ de los estudiantes presentan un nivel de conocimientos medio sobre el aborto inducido y una actitud de indiferencia hacia el aborto inducido; el $18.0 \%$ de estudiantes presentan un nivel de conocimiento bajo sobre el aborto inducido y una actitud de aceptación del aborto, mientras que el $11.7 \%$ de los estudiantes presentan un nivel de conocimientos alto sobre el aborto inducido y una actitud de rechazo hacia el aborto inducido. Se aprecia que mientras más alto el conocimiento mayor actitud de rechazo hacia el aborto y mientras más bajo es el nivel de conocimientos mayor actitud de aceptación hacia el aborto inducido.

Tabla 2

Prueba de chi cuadrado entre conocimientos y actitudes hacia el aborto inducido en estudiantes de la Gran Unidad Escolar Las Mercedes - 2015.

\begin{tabular}{lccc}
\hline & Valor & gl & Sig. asintótica (bilateral) \\
\hline Chi-cuadrado de Pearson & $39.408(a)$ & 4 & .000 \\
Razón de verosimilitudes & 43.836 & 4 & .000 \\
Asociación lineal por lineal & 31.795 & 1 & .000 \\
N de casos válidos & 128 & & \\
\hline
\end{tabular}

a 0 casillas $(.0 \%)$ tienen una frecuencia esperada inferior a 5 . La frecuencia mínima esperada es 6.34 .

La tabla 2 muestra los estadístico no paramétrico del chi cuadrado, donde se aprecia que existe un valor de sig $=.000$, indicando que existe relación entre el nivel de conocimientos sobre aborto inducido y las actitudes hacia el aborto inducido. 
Grover Eyden Trujillo Mamani -

Efrain Sembrera Farceque

\section{En relación al primer objetivo especifico}

Tabla 3

Conocimiento sobre aborto inducido que presentan los adolescentes del quinto año de educación secundaria de la Gran Unidad Escolar Las Mercedes de la ciudad de Juliaca-2015.

\begin{tabular}{ccccc}
\hline $\begin{array}{c}\text { Nivel de Co- } \\
\text { nocimiento }\end{array}$ & Frecuencia & Porcentaje & $\begin{array}{c}\text { Porcentaje } \\
\text { válido }\end{array}$ & $\begin{array}{c}\text { Porcentaje } \\
\text { acumulado }\end{array}$ \\
\hline Bajo & 30 & 23.4 & 23.4 & 23.4 \\
Medio & 69 & 53.9 & 53.9 & 77.3 \\
Alto & 29 & 22.7 & 22.7 & 100.0 \\
Total & 128 & 100.0 & 100.0 & \\
\hline
\end{tabular}

Fuente: Encuesta de conocimiento a los estudiantes de la I.E. G.U.E.L.M.

La tabla 3 muestra los niveles de conocimiento sobre aborto inducido que presentan los estudiantes del quinto año de educación secundaria de la Institución educativa Gran Unidad Escolar Las Mercedes de la ciudad de Juliaca, donde se aprecia que el $53.9 \%$ presentan un nivel medio de conocimientos sobre aborto inducido, es decir que el conocimiento sobre los conceptos de aborto, así como las causas y consecuencias es de forma parcial; el $23.4 \%$ de los estudiantes presenta un nivel bajo (sólo acertaron hasta tres respuestas correctas de las 14 preguntas) y finalmente sólo el $22.7 \%$ de los estudiantes presenta un nivel alto de conocimientos, es decir, que este grupo de estudiantes conocen los conceptos de aborto inducido, así como las causas y consecuencias del mismo.

\section{En relación al segundo objetivo especifico}

Tabla 4

Actitudes hacia el aborto inducido que presentan los adolescentes del quinto año de educación secundaria de la Gran Unidad Escolar Las Mercedes de la ciudad de Juliaca - 2015.

\begin{tabular}{ccccc}
\hline Actitud & Frecuencia & Porcentaje & $\begin{array}{c}\text { Porcentaje } \\
\text { válido }\end{array}$ & $\begin{array}{c}\text { Porcentaje } \\
\text { acumulado }\end{array}$ \\
\hline De aceptación & 55 & 43.0 & 43.0 & 43.0 \\
De indiferencia & 45 & 35.2 & 35.2 & 78.1 \\
De rechazo & 28 & 21.9 & 21.9 & 100.0 \\
Total & 128 & 100.0 & 100.0 & \\
\hline
\end{tabular}

Fuente: Encuesta de actitudes a los estudiantes de la I.E. G.U.E.L.M. 
La tabla 4 muestra las actitudes hacia el aborto inducido que presentan los estudiantes del quinto año de educación secundaria de la Institución educativa Gran Unidad Escolar Las Mercedes de la ciudad de Juliaca, se entiende por actitud como el pensamiento favorable o desfavorable hacia el aborto inducido y donde se aprecia que $43.0 \%$ de los adolescentes presentan una actitud de aceptación hacia el aborto inducido, es decir, presentan pensamientos favorables hacia la práctica del aborto inducido; el $35.2 \%$ de los estudiantes presentan una actitud de indiferencia es decir no están ni a favor ni en contra del aborto inducido y finalmente sólo el $21.9 \%$ de los estudiantes presentan una disposición de rechazo hacia tal práctica.

\subsection{Discusión}

La adolescencia es una etapa entre la niñez y la edad adulta que se inicia por los cambios puberales y se caracteriza por profundas transformaciones biológicas, psicológicas y sociales; muchas de ellas generadoras de crisis, conflictos y contradicciones. No es solamente un periodo de adaptación a los cambios corporales, sino una fase de grandes determinaciones hacia una mayor independencia psicológica y social (Palomino, 2009).

Los resultados del nivel de conocimientos en la presente investigación evidencian que el $53.9 \%$ de los estudiantes presentan un nivel medio y el $23.4 \%$ es bajo, es decir que los conocimientos que poseen son erróneos. La información que poseen los adolescentes sobre el conocimiento sexual, sus normas y valores es falsa, lo que los hace doblemente expuestos, por los erróneos conceptos que reciben de sus pares, predisponiéndolos a desarrollar conductas sexuales de riesgo.

Por lo anteriormente mencionado podemos afirmar que los estudiantes del quinto año de educación secundaria en promedio tienen nivel de conocimientos "medio" sobre el aborto inducido, por lo que se considera que los adolescentes se encuentran en un proceso de adquisición de conocimientos sobre la problemática, por lo que es necesario en esta etapa de vida incrementar la información sobre la sexualidad y las consecuencias del aborto en los adolescentes, con la finalidad de que adopten conductas sexuales saludables.

Los resultados hallados en la presente investigación guardan relación con los reportados por Delgado (2008, citado en Navarro y López, 2012) donde refiere que la mayoría de los adolescentes presentan un nivel medio (42.8\%) de conocimientos sobre sexualidad, dentro de los cuales se halla el tema del aborto, seguido de los niveles bajo (35.2\%) y alto (22\%) respectivamente, además menciona que los adolescentes desconocen las prácticas sexuales riesgosas y la 
Grover Eyden Trujillo Mamani -

Efrain Sembrera Farceque

manifestación de sus impulsos sexuales se encuentra presente mediante besos, caricias y relaciones cóitales, que traen como consecuencia los embarazos precoces y no deseados, abortos, ITS y otros.

Estudios reportados por Gutiérrez, Safora, y Rodríguez (2009) sobre los conocimientos acerca del aborto, refieren que el 74\% de los adolescentes entre 15 y 19 años de edad presentan conocimientos correctos y el $26 \%$ de los adolescentes presentan conocimientos incorrectos, estos resultados guardan relación con los hallados en la presente investigación donde el $23.4 \%$ de los adolescentes del quinto año de educación secundaria presentan un nivel bajo, es decir que sus conocimientos son incorrectos y el $76.6 \%$ de los adolescentes presentan un nivel de conocimientos medio y alto, es decir que presentan conocimientos correctos y parcialmente correctos acerca del aborto.

Palomino (2009) también refiere que la mayoría de adolescentes (68.75\%) presentan un nivel medio de conocimientos sobre el aborto inducido, guardando relación con los datos presentados en la presente investigación.

En relación a las actitudes hacia el aborto inducido en la población de estudio, el mayor porcentaje de estudiantes presentan una actitud de aceptación hacia el aborto inducido (43.0\%), seguido del $35.2 \%$ con una actitud de indiferencia, estos datos se asemejan con los reportados por Palomino (2009) en la región Lima, donde la mayoría de adolescentes presentan una actitud de indiferencia (66.06\%), seguido de la actitud de aceptación (21.82\%) y finalmente reporta una actitud de rechazo (12.12\%), los datos difieren de los reportados en la presente investigación, y estos podrían ser explicados por las estadísticas que se registran a nivel de la Región Puno donde la Red de Salud de San Román ocupa el primer lugar en presentar abortos y del $100 \%$ de abortos presentados en San Román, la mitad corresponden a menores de edad (Los Andes, 2013).

Así mismo en la Región de Puno, Leoncio Aguilar Hilari, responsable del servicio de obstetricia del hospital Carlos Monge Medrano de Juliaca, provincia de San Román (región Puno) dio a conocer la cifra indicando que de cada 10 embarazos, tres terminan en abortos (RPP, 2015).

Palomino (2009) señala que la actitud es una disposición psicológica adquirida y organizada a través de la experiencia que suscita al individuo a reaccionar de manera característica frente a determinados objetos, personas, situaciones, es decir una disposición de aceptación o rechazo que se expresa mediante el comportamiento. La actitud que poseemos hacia personas o cosas es indiferente por falta de conocimientos y sostiene que entre los factores más importante en la formación de las actitudes son las experiencias personales, así también influye la familia, los compañeros, la educación, los incidentes críticos. 
Los resultados hallados demuestran que existe relación entre el nivel de conocimientos y actitudes hacia el aborto inducido, los cuales guardan relación con los hallados por Palomino quien realizó la investigación en una población de adolescentes entre 12 y 18 años de edad (2009).

La adolescencia es considerada un periodo de crisis en el ciclo de vida de la persona, debido a que se generan diversos cambios (físicos y emocionales); el adolescente durante este periodo va fortaleciendo su personalidad asimismo evidencia inseguridad, desconfianza y confusión, sobre su sexualidad, predisponiéndolo al desarrollo de conductas sexuales de riesgo una de ellas el aborto.

La adolescencia es una etapa entre la niñez y la edad adulta que se inicia por los cambios puberales y se caracteriza por profundas transformaciones biológicas, psicológicas y sociales; muchas de ellas generadoras de crisis, conflictos y contradicciones. No es solamente un periodo de adaptación a los cambios corporales, sino una fase de grandes determinaciones hacia una mayor independencia psicológica y social, por lo que la información que puedan poseer, sobre el conocimiento sexual, es de vital importancia para la toma de decisiones, y adoptar conductas sexuales saludables asociados a principios ético morales cristianos.

La sexualidad es algo positivo, que hace agradables nuestras vidas. Por lo tanto, lo primero para ejercer nuestra sexualidad como una oportunidad de placer y bienestar, es asumir que nuestro cuerpo y nuestros deseos sexuales son digno motivo de alegría y no una manifestación de pecado o un motivo de castigo. Nuestra sexualidad tiene componentes biológicos, psicológicos, sociales y espirituales. En especial los elementos sociales van construyendo nuestra sexualidad de acuerdo a las normas que en nuestra sociedad aprueban determinados comportamientos y rechazan otros (Navarro \& López, 2012).

\section{Conclusiones}

Primera.- A un nivel de significancia del $5 \%$, existe correlación significativa entre el nivel de conocimientos sobre aborto inducido y las actitudes hacia el aborto inducido en los estudiantes del quinto año de educación secundaria de la I.E. Gran Unidad Escolar Las Mercedes de la ciudad de Juliaca.

Segunda.- El mayor porcentaje de estudiantes del quinto año de educación secundaria de la I.E. Gran Unidad Escolar Las Mercedes de la ciudad de Juliaca, presentan un nivel medio $(53.9 \%)$ de conocimientos sobre el aborto 
Grover Eyden Trujillo Mamani -

Efrain Sembrera Farceque

inducido, seguido del nivel bajo (23.4\%) y finalmente solo $(22.7 \%)$ presenta un nivel alto de conocimientos sobre el aborto.

Tercera.- El mayor porcentaje de estudiantes del quinto año de educación secundaria de la I.E. Gran Unidad Escolar Las Mercedes de la ciudad de Juliaca, presentan una actitud de aceptación (43.0\%) hacia el aborto inducido, seguido por las actitud de indiferencia (35.2\%) y finalmente solo (21.9\%) presenta una actitud rechazo hacia el aborto inducido.

Cuarta.- Mientras más alto el conocimiento mayor actitud de rechazo hacia el aborto inducido y mientras más bajo es el nivel de conocimientos mayor actitud de aceptación hacia el aborto inducido.

\section{Recomendaciones}

Primera.- Concientizar al personal de los establecimientos de salud encargados de la Estrategia de Salud del Adolescente, diseñar e implementar talleres sobre conductas o comportamientos sexuales; y realizar sesiones educativas de salud preventiva sobre el aborto y sus consecuencias, planificación familiar saludable, ITS, VIH/SIDA, para mejorar los conocimientos de los adolescentes y disminuir los casos de aborto inducido y llevar una vida sexual segura y responsable.

Segunda.- Capacitar al personal docente a través de la Dirección General de la I.E. Gran Unidad Escolar Las Mercedes, en coordinación con el personal de salud, sobre temas como: la adolescencia, sexualidad, comportamientos sexuales, realizar talleres de sensibilización sobre las consecuencias del aborto inducido y generar cambio en las actitudes. Así mismo realizar convenio con la E.P. de Enfermería de la Universidad Peruana Unión, para que realicen prácticas comunitarias y a través de ello brindar educación sobre sexualidad saludable.

Tercera.- A la Red de San Román a través de la Dirección Regional de Salud - Puno, confeccionar trípticos sobre el aborto inducido y consecuencias con la finalidad de distribuirlos en la comunidad educativa de la región Puno para elevar el nivel de conocimientos frente a dicho tema.

Cuarta.- A la Dirección General de Salud, crear alianzas estratégicas entre los centros de salud, instituciones educativas a través de la UGEL, y los municipios de la región, para enfatizar la educación y la sensibilización sobre temas de sexualidad, que incluyan temas como: el embarazo en adolescente, el aborto inducido y sus complicaciones, las ITS, VIH/SIDA, entre otros. 
Grover Eyden Trujillo Mamani Universidad Peruana Unión, Perú email: trujillogrover.e17@gmail.com

Recibido: 14 de octubre de 2015 Aceptado: 20 de diciembre de 2015

\section{Referencias}

Acho, S., Camposano, D., \& Canova, E. (2005). Aborto en adolescentes. Experiencia en el Hospital Nacional Cayetano Heredia. Revista Peruana de Ginecología y Obstetricia, 51(2), 100-104.

Barra, E. (1998). Psicología Social. Chile: Universidad de Concepción.

Enríquez, B., Bermúdez, R., Puentes, E. M., \& Jiménez, M. C. (2010). Comportamiento del aborto inducido en la adolescencia. Revista Cubana de Obstetricia y Ginecología, 36(2), 145-159.

Domínguez, P., \& Espeso, E. (2002). El conocimiento metacognitivo y su influencia en el aprendizaje motor. Internacional de Medicina y Ciencias de la Actividad Física y el Deporte, 2(4), 5968. Retrieved from http://cdeporte.rediris.es/revista/revista4/artmeta.htm

Gutiérrez-Ramos, M. (2005). El aborto. Atención humanizada de sus complicaciones como estrategia para la disminución de la mortalidad materna. Acta Médica Peruana, XXII(2), 80-85.

Hernandez, R., Fernández, C., \& Baptista, P. (2010). Metodología de la investigación (Quinta ed.). México: McGraw-Hill.

Inostroza, O. E., \& Quezada, C. A. (2012). El aborto terapéutico y su regulación en Chile: Derecho comparado y evolución histórica. Tesis para optar al grado de Licenciado en Ciencias Jurídicas y Sociales, Universidad de Chile, Departamento de derecho penal, Santiago, Chile.

Mendoza, V. E., \& Palacios, J. E. (2011). Abortos inducidos en el Hospital Dr. Verdi Cevallos Balda febrero - octubre del 2011. Tesis de grado previa a la optención del título de Médico Cirujano, Universidad Técnica de Manabí, Carrera de Medicina, Manabí, Ecuador.

Mendoza, W., \& Subiria, G. (2013). El embarazo adolescente en el Perú: Situación actual e implicancias para las políticas públicas. Rev Peru Med Exp Salud Pública, 30(3), 471-479.

Motta, L. (2009). Módulo de psicología social. Bucaramanga - Colombia: Universidad Nacional Abierta y a Distancia.

Narvaja, P., \& Canavessi, J. J. (2010). Problemas sobre el conocimiento. Universidad del Salvador.

Neira, J. (2002). Aborto, aspectos clínicos y epidemiológicos. Revista de Estudios Médico Humanísticos, 6(6), 77-82.

OMS. (2014, Marzo). Prevención del aborto peligroso. Retrieved Enero 25, 2014, from Centro de prensa: http://www.who.int/mediacentre/factsheets/fs388/es/

Orduz, R., Vallejo, M. E., \& Ayala, L. (2012). Aprender y educar con las tecnologías del siglo XXI. Bogotá, Colombia: Colombia Digital.

Palomino, M. L. (2009). Qué saben sobre el aborto inducido y que actitudes se evidencian en los adolescentes I.E. Micaela Bastidas. Tesis para optar el título profesional de Licenciada en Enfermería, Universidad Nacional Mayor de San Marcos, Facultad de Medicina Humana.

Paredes, N. (2014, Enero 7). RPP. Retrieved Enero 14, 2015, from Erá Tabú: http://www.rpp.com. pe/2014-01-07-el-aborto-y-sus-consecuencias-fisicas-y-psicologicas-noticia_660191. html

Revista Apunt. Univ.

VOLUMEN VI • NÚMERO 1 p. $47-60$ 


\section{Grover Eyden Trujillo Mamani - Efrain Sembrera Farceque}

Radio Programas del Perú. (2011, Junio 17). RPP Nacional. Retrieved from Arequipa es la tercera ciudad del país donde se registran más abortos: http://www.rpp.com.pe/2011-06-17-arequipaes-la-tercera-ciudad-del-pais-donde-se-registran-mas-abortos-noticia_376493.html\#

Ribeiro da Fonseca, S., Barbosa, M. A., Pinto, M. C., \& Moura, D. (2013, Julio - Agosto). Experiencia de mujeres con el aborto provocado en la adolescencia por imposición de la madre. Revista Latinoamericana Enfermagen, 21(4), 1-8.

Schorge, J., Schaffer, J., Halvorson, L., Hoffman, B., Bradshaw, K., \& Cunningham, F. (2009). Williams Gínecologia. México: McGraw-Hill.

Valderrama Mendoza, S. (2002). Pasos para elaborar Proyectos y Tesis de Investigación Científica. Lima: San Marcos.

Villalobos, L. (2011). Actitud de los estudiantes de educación media ante el método científico. Trabajo de grado presentado como requisito para optar el Título de Magíster Scientiarium en Enseñanza de la Biología, Universidad del Zulia, Facultad de Humanidades y Educación, Macaraibo, Venezuela. 